POSTER

\title{
The Effect of Virgin Coconut Oil (VCO) on Physiological Halitosis. A Randomized Controlled Pilot Trial
}

Zurainie Abllah¹, Siti Khadijah Shahnon², Nurain Najihah Nuawi², Yunita Dewi Ardini ${ }^{1}$

${ }^{1}$ Department of Paediatric Dentistry \& Dental Public Health, Kulliyyah of Dentistry, International Islamic University Malaysia, Kuantan Pahang

${ }^{2}$ Kulliyyah of Dentistry, International Islamic University Malaysia, Kuantan Pahang.

Introduction: VCO oil pulling therapy has been used as a remedy for oral malodour in traditional Indian folk.Main objective for this research is to study on the effect of VCO oil pulling on physiological halitosis among IIUM Kuantan Campus students. The specific objectives are to evaluate the effect of VCO oil pulling on physiological halitosis and to compare the efficacy of VCO with conventional mouthwash in reducing physiological halitosis. Materials and method: 20 convenient samples were selected based on inclusion and exclusion criteria and divided into two groups, mouthwash and VCO based on single blind randomized controlled trial. Subjects required swishing oil pulling (group A) or rinsing with mouthwash (group B). Breathing analysis was recorded during pre-assessment, post assessment (on day 1short term and day 14-long term) using organoleptic 1, organoleptic 2 (selfassessment) and Tanita device. The results were analysed using paired t-test in SPSS version 22. IREC approval: 2018-061. Result: There were significant difference between pre and post assessment of mouthwash in short term $(p<0.001)$ and long term ( $p=0.002)$ period of usage and between group VCO pre and post assessment in short term $(p=0.001)$. However, there was no significant $(p<0.05)$ between group of VCO and mouthwash. Conclusion: VCO has potential to reduce physiological halitosis in immediate observation after the oil pulling procedure. VCO oil pulling and gargling with commercial mouthwash had no significant difference in reducing physiological halitosis. 